\title{
Inflation with non-minimal coupling: Metric vs. Palatini formulations
}

\author{
Florian Bauer ${ }^{a}$, Durmuş A. Demir ${ }^{\mathrm{a}, \mathrm{b}, *}$ \\ a Deutsches Elektronen-Synchrotron, DESY, 22607 Hamburg, Germany \\ b Department of Physics, Izmir Institute of Technology, TR35430 Izmir, Turkey
}

\section{A R T I C L E I N F O}

\section{Article history:}

Received 30 March 2008

Accepted 10 June 2008

Available online 14 June 2008

Editor: M. Cvetič

\begin{abstract}
A B S T R A C T
We analyze non-minimally coupled scalar field theories in metric (second-order) and Palatini (first-order) formalisms in a comparative fashion. After contrasting them in a general setup, we specialize to inflation and find that the two formalisms differ in their predictions for various cosmological parameters. The main reason is that dependencies on the non-minimal coupling parameter are different in the two formalisms. For successful inflation, the Palatini approach prefers a much larger value for the non-minimal coupling parameter than the Metric approach. Unlike the Metric formalism, in Palatini, the inflaton stays well below the Planck scale whereby providing a natural inflationary epoch.
\end{abstract}

(C) 2008 Elsevier B.V. Open access under CC BY license.

\section{Introduction}

Scalar fields play a fundamental role in various physical phenomena ranging from electroweak symmetry breaking to cosmological inflation. Though no fundamental scalar has been observed to date, they are an indispensable part of the theoretical landscape: They can trigger breakdown of gauge symmetries to induce particle masses, they can facilitate inflation to explain shortcomings of standard cosmology, they can make up quintessence to explain amount of dark energy in the universe, and they can act as source of the non-baryonic dark matter in the universe.

Given this rather widespread role of scalar fields at various energy scales, it is of physical relevance to examine their dynamics in geometries more general than general relativity (based on Metric formulation or second-order formalism). Indeed, huge discrepancy between experimental and quantum-theoretic values of the dark energy, absence of any direct observation of dark matter particles though a considerable amount is needed for cosmological concordance, and unknown nature of gravitational dynamics at small and large distances all motivate consideration of a more general geometrical arena than general relativity. Given that there is no unique way of generalization, one possibility, among many one can consider, is to exploit certain features of the affine spaces. The essential observation is that the affine connection and metric tensor are a priori independent geometrical variables, and if they are to exhibit any relationship it must arise from dynamical equations a posteriori. This very setup, the so-called Palatini formulation or first-order formalism [1], does not necessarily admit the Levi-

\footnotetext{
* Corresponding author at: Department of Physics, Izmir Institute of Technology, TR35430 Izmir, Turkey.

E-mail address: durmus.demir@desy.de (D.A. Demir).
}

Civita connection if the matter sector depends explicitly on the affine connection $[2,3]$.

This work is devoted to a comparative analysis of the dynamics of non-minimally coupled scalar fields in Metric and Palatini formulations. It will be shown that the two formalisms generally yield different answers for both metric tensor and scalar fields provided that non-minimal coupling of the scalar field to curvature scalar does not vanish. The analysis will be targeting, for definiteness and concreteness, scalar field theories which can facilitate both electroweak symmetry breaking and inflation.

In the next section we give a comparative discussion of Palatini and Metric formalisms in a general setting in which a scalar field interacts non-minimally with the spacetime curvature. In Section 3 we analyze inflation with non-minimally coupled scalar field, and contrast predictions of the two formalisms by computing various inflationary parameters. In Section 4 we conclude.

\section{Metric vs. Palatini formulations: Generalities}

In this section, we give a brief discussion of the Metric and Palatini formulations in a comparative fashion within a general setup.

An affine connection, whose components to be symbolized by $\Gamma_{\alpha \beta}^{\lambda}$, governs parallel transport of tensor fields along a given curve in spacetime, and parallel transport around a closed curve, after one complete cycle, results in a finite mismatch if the spacetime is curved. This curving is uniquely determined by the Riemann tensor $\mathbb{R}_{\alpha \nu \beta}^{\mu}(\Gamma)$ whose contraction $\mathbb{R}_{\alpha \beta}(\Gamma) \equiv \mathbb{R}_{\alpha \mu \beta}^{\mu}(\Gamma)$ gives the Ricci tensor. ${ }^{1}$

\footnotetext{
1 The affine connection determines not only the curving but also the twirling of the spacetime. The latter is encoded in the torsion tensor $[2,4]$, and it is discarded from the analysis here by considering a symmetric connection $\Gamma_{\alpha \beta}^{\lambda}=\Gamma_{\beta \alpha}^{\lambda}$ only.
} 
The spacetime gets further structured by the notion of distance if it is endowed with a metric tensor $g_{\mu \nu}$ representing clocks and rulers. The connection coefficients and metric tensor are fundamentally independent quantities. They exhibit no a priori known relationship, and if they are to have any it must derive from a additional constraint or geometrodynamics [1].

For explicating differences and similarities between the Metric and Palatini approaches it proves useful to focus on a generic action

$\mathcal{S}=\int \mathrm{d}^{4} x \sqrt{-g}\left\{-\frac{1}{2} M^{2} g^{\mu v} \mathbb{R}_{\mu \nu}(\Gamma)+\mathcal{L}_{\mathrm{m}}(\varphi, \Gamma, g)\right\}$

comprising gravitational (taken to have Einstein-Hilbert form) and material (parameterized by the Lagrangian $\mathcal{L}_{\mathrm{m}}(\varphi, \Gamma, g)$ involving the matter fields $\varphi$, metric tensor $g_{\alpha \beta}$, and possibly also the affine connection $\Gamma_{\alpha \beta}^{\lambda}$ ) sectors. The mass parameter $M$ may or may not be identical to the fundamental scale of gravity $M_{\mathrm{Pl}}=\left(8 \pi G_{N}\right)^{-1 / 2}$ since $\mathcal{L}_{\mathrm{m}}(\varphi, \Gamma, g)$ can feed further contributions to Newton's constant.

The Metric formulation refers to replacing $\Gamma$ in (1) by $\bar{\Gamma}$ where

$\bar{\Gamma}_{\alpha \beta}^{\lambda}=\frac{1}{2} g^{\lambda \rho}\left(\partial_{\alpha} g_{\beta \rho}+\partial_{\beta} g_{\rho \alpha}-\partial_{\rho} g_{\alpha \beta}\right)$

is the Levi-Civita connection. Having this constraint, the metric tensor $g_{\alpha \beta}$ and matter fields $\varphi$ remain as the only independent dynamical variables, and extremization of the action (1) with respect to them yields the associated equations of motion. One notes, however, that the curvature tensor involves second derivatives of the metric tensor, and thus, cancellation of the associated surface terms requires enhancement of (1) by an extrinsic curvature contribution.

The Palatini formulation refers to keeping the metric, the connection and matter fields as independent dynamical variables in (1). Therefore, the affine connection is not a predetermined quantity at all; it takes the form that dynamics requires. Moreover, the action involves only the first derivatives of the connection, and hence, there arises no surface term to be cancelled by an extrinsic curvature contribution.

An important feature of the Palatini formulation is that if $\mathcal{L}_{\mathrm{m}}(\varphi, \Gamma, g)$ is independent of $\Gamma_{\alpha \beta}^{\lambda}$ then the equation of motion for the connection

$\nabla_{\lambda}^{\Gamma}\left(\sqrt{-g} g^{\alpha \beta}\right)=0$

uniquely returns $\Gamma=\bar{\Gamma}$. Therefore, the Levi-Civita connection arises dynamically with no need to extrinsic curvature [1]. Nevertheless, this dynamical relaxation to the Metric formulation gets spoiled immediately if $\mathcal{L}_{\mathrm{m}}(\varphi, \Gamma, g)$ depends on the affine connection $\Gamma_{\alpha \beta}^{\lambda}$ explicitly. Indeed, in this case variation of the matter action with respect to the connection gives additional contributions to (3) whereby causing $\Gamma_{\alpha \beta}^{\lambda}$ to deviate from $\bar{\Gamma}_{\alpha \beta}^{\lambda}$ as a function of the matter fields. ${ }^{2}$

The matter sector consists of all kinds of fields whose interactions are encoded in $\mathcal{L}_{\mathrm{m}}(\varphi, \Gamma, \mathrm{g})$. In general, at least at the renormalizable level, Lagrangians of vector fields do not involve the connection (yet, see [3]). Fermion fields, however, do explicitly depend on $\Gamma$ via the spin connection in their kinetic terms. Therefore, the equation of motion (3) gets generically modified by the fermion sector. However, if needed, these contributions can be compensated by modifying the fermion Lagrangian via contact

\footnotetext{
2 The setup of (1) is actually not the most general one. Indeed, the EinsteinHilbert term can be replaced by a more general structure containing a generic function of curvature invariants. In this case the deviation of $\Gamma$ from $\bar{\Gamma}$ can be more involved; in particular, the equation of motion (3) generalizes to contain new structures involving derivatives of $\Gamma$ itself.
}

terms quadratic in the torsion $[2,4]$. In this work, following this observation, fermion contributions to the affinity $\Gamma$ will be discarded. Coming to scalar fields, like vector fields, they are also independent of the connection when minimally coupled. However, they develop a direct dependence on the connection, already at the renormalizable level, by direct coupling to the curvature scalar. Indeed, the Lagrangian of a scalar field $\phi$

$\mathcal{L}_{\mathrm{m}}=\frac{1}{2} g^{\mu \nu} \partial_{\mu} \phi \partial_{\nu} \phi-\frac{1}{2} \zeta \phi^{2} g^{\mu \nu} \mathbb{R}_{\mu \nu}(\Gamma)-V(\phi)$

generically involves a nontrivial coupling to the curvature scalar ${ }^{3}$ via a dimensionless parameter $\zeta$. This coupling can take any real value. $^{4}$

Given the non-minimally coupled scalar field in (4), the equation of motion of $\Gamma$ in (3) changes to

$\nabla_{\lambda}^{\Gamma}\left(\left(M^{2}+\zeta \phi^{2}\right) \sqrt{-g} g^{\alpha \beta}\right)=0$

with the solution

$\Gamma_{\alpha \beta}^{\lambda}=\bar{\Gamma}_{\alpha \beta}^{\lambda}+\delta_{\alpha}^{\lambda} \partial_{\beta} \omega(\phi)+\delta_{\beta}^{\lambda} \partial_{\alpha} \omega(\phi)-g_{\alpha \beta} \partial^{\lambda} \omega(\phi)$

where

$\omega(\phi)=\ln \sqrt{\frac{M^{2}+\zeta \phi^{2}}{M_{\mathrm{Pl}}^{2}}}$

with the normalization scale $M_{\mathrm{Pl}}$ being chosen just for convenience. $^{5}$ It is this very difference of $\Gamma$ from $\bar{\Gamma}$ that makes nonminimally coupled scalar fields behave differently in Metric and Palatini formulations.

Another way of seeing differences in scalar field dynamics in Metric and Palatini approaches comes by changing the frame. Indeed, the action (1) with the scalar field Lagrangian (4) corresponds to the Jordan frame. However, though there is no obvious reason for preferring one over the other, it is possible to analyze the whole dynamics in the Einstein frame, too. To do this, it suffices to transform the metric as [7]

$g_{\mu \nu} \rightarrow e^{-2 \omega(\phi)} g_{\mu \nu}$

after which the coefficient in front of $g^{\mu \nu} \mathbb{R}_{\mu \nu}(\Gamma)$ becomes $\frac{1}{2} M_{\mathrm{Pl}}^{2}$, as it would be for a minimally-coupled scalar field. This transformation gives rise to different dynamics for the scalar field in Palatini and Metric approaches since:

- In the Palatini case $\mathbb{R}_{\mu \nu}(\Gamma)$ does not depend on the metric and therefore does not change under (8). In the Metric case, the action (1) contains $\mathbb{R}_{\mu v}(\bar{\Gamma})$ and thus the metric from the scratch, and it does change with (8).

- The kinetic term of the scalar field in the Palatini case only gets multiplied by $e^{-2 \omega(\phi)}$ under (8). In the Metric case, however, in addition to this rescaling there arises an additional contribution to the kinetic term from the transformation of $\mathbb{R}_{\mu v}(\bar{\Gamma})$ itself.

- In the Palatini case, since the coefficient in front of $g^{\mu \nu} \mathbb{R}_{\mu \nu}(\Gamma)$ is just $M_{\mathrm{Pl}}^{2} / 2$ the affinity $\Gamma$ reduces to the Levi-Civita one (2). Consequently, the difference between the two formalisms lies in the matter sector wherein (8), together with (7), induces different dynamics in Metric and Palatini cases.

\footnotetext{
3 Except for Goldstone bosons, such a coupling generically exists for all scalar fields [5].

4 In the Metric formulation, for $\zeta=1 / 6$ the action for a scalar field gains local conformal invariance [6]. This property does not need to hold in the Palatini formulation where the curvature tensor is intact to transformations on the metric tensor.

5 This new scale must to be introduced here since $M_{\mathrm{Pl}}$ is the true fundamental scale of gravity in the Einstein frame.
} 
Several features of the two formalisms mentioned above hold for a generic scalar field theory. Indeed, the scalar field might be the one that triggers one of those phase transitions in the history of the universe, might be the inflaton, might be quintessence or might be some other one needed for some specific purpose. What is important is that the spacetime evolutions of the scalar field and the metric are different in the two approaches.

In the next section we provide an explicit case study by analyzing early inflation with a specific self-interaction potential $V(\phi)$ that can, though not essential at all for the inflationary regime, also facilitate the electroweak symmetry breaking in small $\phi$ regime.

\section{Inflation: Metric vs. Palatini formulations}

The idea of inflation has ever been the most viable framework for understanding several shortcomings of the standard big bang cosmology [8]. During the inflationary epoch the total energy density of the universe is dominated by that of the vacuum, and the scale factor of the universe grows exponentially $a(t) \sim e^{H t}, H$ being the Hubble rate. If this exponential expansion continues for a time interval $\delta t \sim N / H(N \gtrsim 60)$ then a small causally connected patch gets magnified sufficiently to explain the observed flatness, isotropy, and homogeneity of the universe [8,9]. In spite of these observationally confirmed advantages, inflationary models suffer from problems associated with spoiling of the flatness (by quantum corrections) and super-Planckian values that the inflaton field takes $[10,11]$ (for a recent discussion of the naturalness problem in inflationary models, see [12]).

In what follows, we will study inflation in Metric and Palatini formulations in a comparative fashion, and confront them with each other as well as with the cosmological observations. Meanwhile, we will also discuss naturalness problems encountered in inflationary models [12] within these two formalisms. The procedure that will be outlined below is valid for any inflationary model with its defining inflaton potential $V(\phi)$; however, for concreteness, we will consider a non-minimally coupled scalar field as in (4) such that its potential $V(\phi) \propto\left(\phi^{2}-v^{2}\right)^{2}$ can facilitate both electroweak symmetry breaking at small $\phi$ and inflation at large $\phi$. In fact, in [13] (see also [14]) it was shown that one can indeed have a period of early inflation with the standard model Higgs sector alone by allowing for a non-minimal coupling between the Higgs field and the curvature scalar (see also [15] for earlier discussions of inflation with non-minimal coupling). The analysis below is rather generic and general yet various results will be presented in parallel to [13] so as to allow for quantitative comparison.

The setup of the inflationary scenario we discuss is formed by the scalar field action (4) replaced in the general action (1)

$$
\begin{aligned}
\mathcal{S}= & \int \mathrm{d}^{4} x \sqrt{-g}\left[-\frac{M^{2}+\zeta \phi^{2}}{2} g^{\mu \nu} \mathbb{R}_{\mu \nu}(\Gamma)+\frac{1}{2} g^{\mu \nu} \partial_{\mu} \phi \partial_{\nu} \phi\right. \\
& \left.-V(\phi)+\mathcal{L}_{\mathrm{m}}\right]
\end{aligned}
$$

where we have separated the rest of the fields plus their interactions with $\phi$ (Yukawa couplings, for instance) by packing them into $\mathcal{L}_{\mathrm{m}} \equiv \mathcal{L}_{\mathrm{m}}(\phi, \varphi, g)$. For the self-interaction potential we take

$V(\phi)=\frac{1}{4} \lambda\left(\phi^{2}-v^{2}\right)^{2}$

which, by construction, facilitates spontaneous symmetry breaking at $\langle\phi\rangle=v$, and grows like $\phi^{4}$ at large $\phi$.

It is convenient to switch from Jordan frame to Einstein frame in which Newton's constant has its usual meaning in general relativity. To do this we perform the transformation of metric in (8) where the function $\omega(\phi)$ is related to the scalar field via relation (7). This transformation procedure gives

$$
\begin{aligned}
\mathcal{S}_{\mathrm{M}}= & \int \mathrm{d}^{4} x \sqrt{-g}\left[-\frac{M_{\mathrm{Pl}}^{2}}{2} g^{\mu \nu} \mathbb{R}_{\mu \nu}(\bar{\Gamma})\right. \\
& +\frac{1}{2}\left[e^{-2 \omega(\phi)}+e^{-4 \omega(\phi)} \frac{6 \zeta^{2} \phi^{2}}{M_{\mathrm{Pl}}^{2}}\right] \partial_{\mu} \phi \partial^{\mu} \phi \\
& \left.+e^{-4 \omega(\phi)}\left[-V(\phi)+\mathcal{L}_{\mathrm{m}}\left(\phi, \varphi, e^{-2 \omega(\phi)} g\right)\right]\right]
\end{aligned}
$$

in the Metric formalism. One notices that, transformation property of the Ricci tensor $\mathbb{R}_{\mu \nu}(\bar{\Gamma})$ under (8) generates, through nonminimal coupling, an extra contribution to the kinetic term proportional to $6 \zeta^{2} \phi^{2}$. In the formalism of [13] it is this term that largely governs the inflationary expansion.

In the Palatini formulation, on the other hand, the Ricci tensor is invariant under (8), and we find for the corresponding action

$$
\begin{aligned}
\mathcal{S}_{\mathrm{P}}= & \int \mathrm{d}^{4} x \sqrt{-g}\left[-\frac{M_{\mathrm{Pl}}^{2}}{2} g^{\mu \nu} \mathbb{R}_{\mu \nu}(\bar{\Gamma})+\frac{1}{2} e^{-2 \omega(\phi)} \partial_{\mu} \phi \partial^{\mu} \phi\right. \\
& \left.+e^{-4 \omega(\phi)}\left[-V(\phi)+\mathcal{L}_{\mathrm{m}}\left(\phi, \varphi, e^{-2 \omega(\phi)} g\right)\right]\right]
\end{aligned}
$$

where we have directly used $\mathbb{R}_{\mu \nu}(\bar{\Gamma})$ since $\Gamma=\bar{\Gamma}$ is automatic because $\Gamma$ appears nowhere else (see the discussions leading to (2) in previous section). Note that varying first the action (9) with respect to $\Gamma$ and subsequently switching to the Einstein frame via (8) leads to the same result.

A comparative glance at (11) and (12) immediately reveals the difference between the two formalisms: the kinetic term of the scalar field (now minimally coupled $\phi$ field). This difference influences the definition of the physical scalar field (to be denoted by $\psi$ hereon) which possesses a canonical kinetic term. Indeed, the physical field $\psi=\psi(\phi)$ follows from

$$
\begin{aligned}
\frac{\mathrm{d} \psi}{\mathrm{d} \phi} & =\sqrt{e^{-2 \omega(\phi)}+6 M_{\mathrm{Pl}}^{2}\left(\omega^{\prime}(\phi)\right)^{2}} \\
& =\frac{M_{\mathrm{Pl}}}{M} \sqrt{\frac{1}{1+\zeta \phi^{2} / M^{2}}+\frac{6 \zeta^{2} \phi^{2} / M^{2}}{\left(1+\zeta \phi^{2} / M^{2}\right)^{2}}}
\end{aligned}
$$

in the Metric formalism. On the other hand, the physical field in the Palatini formalism derives from

$$
\frac{\mathrm{d} \psi}{\mathrm{d} \phi}=\sqrt{e^{-2 \omega(\phi)}}=\frac{M_{\mathrm{Pl}}}{M} \sqrt{\frac{1}{1+\zeta \phi^{2} / M^{2}}}
$$

which differs from (13) by the absence of the second term in radical sign (which is generated by the transformation of $\mathbb{R}_{\mu \nu}(\bar{\Gamma})$ under (8)).

In what follows, we will admit large values of non-minimal coupling $(\zeta \gg 1)$ but we will not identify $M$ with $M_{\mathrm{Pl}}$ from the scratch. We will consider both large and small values of $\psi$ to reveal the potential of the model for both inflation and electroweak symmetry breaking separately in the Metric and Palatini approaches.

Electroweak regime. From (13) and (14) it is clear that

$\psi \simeq \frac{M_{\mathrm{Pl}}}{M} \phi$

in the limit $\phi \sqrt{\zeta} \ll M$. This result is valid for both Metric and Palatini approaches. In this case, the potential of $\psi$ takes the form

$V(\psi) \simeq \frac{1}{4} \lambda\left(\psi^{2}-\frac{M_{\mathrm{Pl}}^{2}}{M^{2}} v^{2}\right)^{2}$

from which its mass and VEV follow to be

$m_{\psi}^{2}=2 \lambda \frac{M_{\mathrm{Pl}}^{2} v^{2}}{M^{2}}, \quad\langle\psi\rangle=\frac{M_{\mathrm{Pl}} v}{M}$. 
This setup can trigger the electroweak symmetry breaking once various mass scales are assigned the correct values. There are essentially two possibilities: (i) If the field $\phi$ is the standard model Higgs field (in unitary gauge) then $v \sim M_{W}$, and thus, $M \sim M_{\mathrm{Pl}}$ for the minimally-coupled $\psi$ field to keep playing the role of Higgs field like $\phi$. (ii) If only the field $\psi$, not $\phi$, is relevant for electroweak symmetry breaking then all one has is the condition $M_{\mathrm{Pl}} v / M \sim M_{W}$. In this case one can take either (ii.a) $v \sim M_{W}$ and $M \sim M_{\text {Pl }}$ or (ii.b) $v \sim m_{v}$ and $M \sim M_{W}$. Both cases, with $m_{v}$ being a typical neutrino mass, lead to the same result that $\psi$ (not $\phi$ ) triggers the electroweak symmetry breaking. The case (ii.b) is particularly interesting as it allows $M \sim M_{W} \ll M_{\mathrm{Pl}}$. It is easy to see that after a suitable rescaling of the fermion fields the Yukawa couplings in $\mathcal{L}_{\mathrm{m}}(\phi, \varphi, g)$ imply standard fermion masses of the order $m_{\psi}$.

Inflationary regime. The inflationary regime refers to large values of the fields. More quantitatively, it is achieved when $\phi \sqrt{\zeta} \gg M$, and in this limit, the Metric formalism yields

$\phi \simeq \frac{M}{\sqrt{\zeta}} \exp \left(\frac{\psi}{\sqrt{6} M_{\mathrm{Pl}}}\right)$

provided that $\zeta \gg 1$. This setup agrees with cosmological observations for

$M \simeq M_{\mathrm{Pl}}, \quad \zeta \simeq 4.9 \times 10^{4} \sqrt{\lambda}$

as has already been derived in [13].

We now focus on the Palatini formulation for an analysis of (12) for examining its predictions for the inflationary regime. First, one notes that, unlike (13), the condition (14) admits direct integration, and hence,

$\phi=\frac{M}{\sqrt{\zeta}} \sinh \left(\frac{\psi \sqrt{\zeta}}{M_{\mathrm{Pl}}}\right)$

exactly. The main difference from (18) is the appearance of $\sqrt{\zeta}$ in the argument of the hyperbolic function. Clearly, the inflationary regime $\sqrt{\zeta} \phi \gg M$ corresponds to taking $\psi \gg M_{\mathrm{Pl}} / \sqrt{\zeta}$. Using (20) the self-interaction potential of $\psi$ can be computed exactly from (12). The large-field limit of the potential

$V(\psi) \simeq \frac{M_{\mathrm{Pl}}^{4} \lambda}{4 \zeta^{2}}\left[1-8\left(1+\frac{\zeta v^{2}}{M^{2}}\right) \exp \left(-\frac{2 \psi \sqrt{\zeta}}{M_{\mathrm{Pl}}}\right)\right]$

is indeed flat, and its flatness and hence relevance for inflation can be quantified via

$$
\begin{aligned}
& \epsilon \equiv \frac{M_{\mathrm{Pl}}^{2}}{2}\left(\frac{V^{\prime}}{V}\right)^{2}=128 \zeta \exp \left(-\frac{4 \psi \sqrt{\zeta}}{M_{\mathrm{Pl}}}\right), \\
& \eta \equiv M_{\mathrm{Pl}}^{2} \frac{V^{\prime \prime}}{V}=-32 \zeta \exp \left(-\frac{2 \psi \sqrt{\zeta}}{M_{\mathrm{Pl}}}\right)
\end{aligned}
$$

which are the slow-roll parameters $[8,13]$ in the Palatini formulation.

The duration of the inflationary period in units of $H^{-1}$ (i.e. the number of $e$-folds) can be directly computed by using (21):

$N=\left.\frac{1}{M_{\mathrm{Pl}}^{2}} \int_{\psi_{\text {end }}}^{\psi_{\text {start }}} \frac{V}{V^{\prime}} \mathrm{d} \psi \simeq \frac{1}{32 \zeta} \exp \left(\frac{2 \psi \sqrt{\zeta}}{M_{\mathrm{Pl}}}\right)\right|_{\psi_{\text {end }}} ^{\psi_{\text {start }}}$

where the inflaton $\psi$ starts with $\psi_{\text {start }}$ and ends with $\psi_{\text {end }} \simeq$ $M_{\mathrm{Pl}} \ln (128 \zeta) /(4 \sqrt{\zeta})$ at which slow-roll regime is spoiled by $\epsilon \simeq 1$. For $\psi_{\text {start }} \gg \psi_{\text {end }}$ from (23) one obtains $32 \zeta N=\exp \left(2 \psi_{\text {start }} \sqrt{\zeta} /\right.$ $\left.M_{\mathrm{Pl}}\right)$. If we further identify $\psi_{\text {start }}$ with the field value at which the
Table 1

Main parameters of the inflationary epoch for $N=62 e$-foldings in the Metric and Palatini formulations

\begin{tabular}{lll}
\hline$\phi \sqrt{\zeta} \gg M$ & Metric [13] & Palatini \\
\hline$\phi(\psi)$ & $\frac{M}{\sqrt{\zeta}} \exp \left(\frac{\psi}{\sqrt{6} M_{\mathrm{Pl}}}\right)$ & $\frac{M}{\sqrt{\zeta}} \sinh \frac{\psi \sqrt{\zeta}}{M_{\mathrm{Pl}}}$ \\
$\zeta$ & $\simeq 4.91 \times 10^{4} \sqrt{\lambda}$ & $\simeq 1.45 \times 10^{10} \lambda$ \\
$\epsilon$ & $\frac{3}{4 N^{2}} \simeq 2.0 \times 10^{-4}$ & $\frac{1}{8 \zeta N^{2}} \simeq 2.2 \times 10^{-15} \lambda^{-1}$ \\
$\eta$ & $-\frac{1}{N} \simeq-0.016$ & $-\frac{1}{N} \simeq-0.016$ \\
$\frac{\psi_{\text {start }}}{M_{\mathrm{Pl}}}$ & $\frac{\sqrt{6}}{2} \ln (4 N / 3) \simeq 5.4$ & $\frac{1}{2 \sqrt{\zeta}} \ln (32 \zeta N) \simeq 1.3 \times 10^{-4} \lambda^{-1 / 2}$ \\
$\frac{\phi_{\text {start }}}{M}$ & $\sqrt{\frac{4 N}{3 \zeta}} \simeq 4.1 \times 10^{-2} \lambda^{-1 / 4}$ & $\sqrt{8 N} \simeq 22$ \\
$\frac{\psi_{\text {end }}}{M_{\mathrm{Pl}}}$ & $\frac{\sqrt{6}}{4} \ln (4 / 3) \simeq 0.18$ & $\frac{1}{4 \sqrt{\zeta}} \ln (128 \zeta) \simeq 5.9 \times 10^{-5} \lambda^{-1 / 2}$ \\
$\frac{\phi_{\text {end }}}{M}$ & $\left(\frac{4}{3 \zeta^{2}}\right)^{1 / 4} \simeq 4.9 \times 10^{-3} \lambda^{-1 / 4}$ & $\left(\frac{8}{\zeta}\right)^{1 / 4} \simeq 4.9 \times 10^{-3} \lambda^{-1 / 4}$ \\
\hline
\end{tabular}

COBE scale enters the horizon when $N \simeq 62$ then the corresponding normalization condition [9]

$\frac{V}{\epsilon} \simeq 2 M_{\mathrm{Pl}}^{4} \lambda \zeta^{-1} N^{2} \simeq\left(0.027 M_{\mathrm{Pl}}\right)^{4}$

leads to

$\zeta \simeq 1.45 \times 10^{10} \lambda$

from which it is clear that the required value of $\zeta$ in the Palatini approach turns out to be approximately five orders of magnitude larger than that of the Metric approach in (19).

At the beginning of the inflationary epoch, the slow-roll parameters at $\psi=\psi_{\text {start }}$ for $N=62 e$-folds read as

$\epsilon=\frac{1}{8 \zeta N^{2}} \simeq 2.2 \times 10^{-15} \lambda^{-1}, \quad \eta=-\frac{1}{N} \simeq-0.016$

which lead to a spectral index of $n=1-6 \epsilon+2 \eta \simeq 0.97$, and a tiny tensor to scalar perturbation ratio $r=16 \epsilon \simeq 10^{-14}$. This very result can be used to readily falsify the Palatini approach in case a significant amount of tensor perturbations are observed. Note that higher-order corrections to $n$ involve the parameters

$\xi^{2} \equiv M_{\mathrm{Pl}}^{4} \frac{V^{\prime} V^{\prime \prime \prime}}{V^{2}}=\frac{1}{N^{2}}$,

$\sigma^{3} \equiv M_{\mathrm{Pl}}^{6} \frac{\left(V^{\prime}\right)^{2} V^{(4)}}{V^{3}}=-\frac{1}{N^{3}}$,

which are significantly smaller than $\eta$. Therefore, they can be safely neglected in our analysis.

For a precise confrontation of the Palatini approach with the Metric one, we find it useful to compare their predictions for various quantities in tabular form. We do this in Table 1 from which we extract a number of important features:

- The $\epsilon$ parameter is (in)dependent of $\zeta$ in the (Metric) Palatini approach. In Palatini, $\epsilon \propto 1 / \zeta$, and it gives rise to an extreme suppression of $\epsilon$ compared to the one in the Metric formalism. One immediate implication of small $\epsilon$ is that the tensorto-scalar ratio of perturbations is approximately 11 orders of magnitude smaller in the Palatini approach than in the Metric approach. This is a testable signature with which one can discard the Palatini approach if a larger amount of tensor perturbations are found.

- The $\eta$ parameter is the same (and much larger than $\epsilon$ in size) for both formalisms; hence, the spectral index $n$ exhibits almost no change with the formalism, and equals $0.968(0.967)$ for the Palatini (Metric) case. Also the parameters $\xi^{2}$ and $\sigma^{3}$ in (27) have the same values in both formalisms.

- The Einstein frame inflaton $\psi$ (having canonical kinetic term) starts with $5.4 \mathrm{M}_{\mathrm{Pl}}\left(1.3 \times 10^{-4} \mathrm{M}_{\mathrm{Pl}}\right)$ and ends with $0.18 \mathrm{M}_{\mathrm{Pl}}$ $\left(5.9 \times 10^{-5} M_{\mathrm{Pl}}\right)$ in the Metric (Palatini) formulation. The initial 
value of $5.4 M_{\mathrm{Pl}}$ is a well-known feature of inflationary models [10-12]. It is this super-Planckian value of $\psi$ that causes a serious naturalness problem in that all higher-dimensional (Planck-suppressed) operators become relevant operators. Contrary to this obviously problematic aspect of the Metric formalism, one immediately observes that the Palatini approach provides an inflationary epoch with $\psi$ staying well below the Planck scale. This sub-Planckian $\psi$-inflaton arises as an important feature of the Palatini approach. Consequently, this approach provides a sensible model of inflation since the inflaton does obviously not escape into the stringy territory.

- The Jordan frame inflaton $\phi$ (having canonical kinetic term plus a direct coupling to curvature scalar) starts with $4.1 \times$ $10^{-2} M(22 M)$ in the Metric (Palatini) approach, and ends with $4.9 \times 10^{-3} M$ in both formalisms. In this case, the Palatini approach seems to require unacceptably large $\phi$ values; however, one notices that the scale $M$, as was discussed while analyzing electroweak symmetry breaking, does not need to be equal to $M_{\mathrm{Pl}}$, in fact, it could be as low as $M_{W}$ without causing any problem with particle masses. In this sense, the Palatini approach turns out to give a natural inflationary epoch for both $\phi$ and $\psi$ inflatons.

These itemized features complete the comparative analysis of inflation in the Metric and Palatini approaches.

\section{Conclusion}

In this work we have performed a comparative analysis of the Metric and Palatini approaches to gravity. Considering nonminimally coupled scalar fields, we have discussed how these two formalisms differ from each other in a general setup in Section 2. In Section 3 we have applied findings of Section 2 to an inflationary setup whose fundamental scalar can trigger both electroweak symmetry breaking and inflation. We have found that both approaches do have observable signatures of their own. A highly important feature is that Palatini approach provides a natural inflation since the inflaton in this formalism stays well below the Planckian regime. Other features, like strong suppression of tensor perturbations, form additional distinctive features of the Pala- tini approach compared to the Metric one. We thus conclude that a non-minimally coupled scalar field in the Palatini formulation gives a sensible inflationary evolution for the early universe.

\section{Acknowledgements}

The work of D.D. was supported by Alexander von HumboldtStiftung Friedrich Wilhelm Bessel-Forschungspreise and by the Turkish Academy of Sciences via GEBIP grant.

\section{References}

[1] A. Palatini, Rend. Circ. Mat. Palermo 43 (1919) 203; A. Einstein, Sitzung-ber Preuss Akad. Wiss. (1925) 414

[2] H. Weyl, Phys. Rev. 77 (1950) 699; S. Deser, C.J. Isham, Phys. Rev. D 14 (1976) 2505; F.W. Hehl, J.D. McCrea, E.W. Mielke, Y. Ne'eman, Phys. Rep. 258 (1995) 1, gr-qc/ 9402012.

[3] S. Deser, Class. Quantum Grav. 23 (2006) 5773, gr-qc/0606006.

[4] F.W. Hehl, P. Von Der Heyde, G.D. Kerlick, J.M. Nester, Rev. Mod. Phys. 48 (1976) 393.

[5] M.B. Voloshin, A.D. Dolgov, Sov. J. Nucl. Phys. 35 (1982) 120, Yad. Fiz. 35 (1982) 213.

[6] A. Iorio, L. O’Raifeartaigh, I. Sachs, C. Wiesendanger, Nucl. Phys. B 495 (1997) 433, hep-th/9607110; D.A. Demir, Phys. Lett. B 584 (2004) 133, hep-ph/0401163.

[7] V. Faraoni, E. Gunzig, P. Nardone, Fund. Cosmic, Phys. 20 (1999) 121, gr-qc 9811047; J.L. Crooks, P.H. Frampton, Phys. Rev. D 73 (2006) 123512, astro-ph/0601051.

[8] A.H. Guth, Phys. Rev. D 23 (1981) 347; A.D. Linde, Phys. Lett. B 108 (1982) 389; A. Albrecht, P.J. Steinhardt, Phys. Rev. Lett. 48 (1982) 1220

[9] D.H. Lyth, A. Riotto, Phys. Rep. 314 (1999) 1, hep-ph/9807278

[10] A.D. Linde, Phys. Lett. B 129 (1983) 177.

[11] K. Freese, J.A. Frieman, A.V. Olinto, Phys. Rev. Lett. 65 (1990) 3233.

[12] N. Arkani-Hamed, H.C. Cheng, P. Creminelli, L. Randall, JCAP 0307 (2003) 003 hep-th/0302034

[13] F.L. Bezrukov, M. Shaposhnikov, Phys. Lett. B 659 (2008) 703, arXiv: 0710.3755 [hep-th].

[14] S.C. Park, S. Yamaguchi, arXiv: 0801.1722 [hep-ph].

[15] V. Faraoni, Phys. Rev. D 53 (1996) 6813, astro-ph/9602111; V. Faraoni, Phys. Rev. D 62 (2000) 023504, gr-qc/0002091; V. Faraoni, Phys. Lett. A 269 (2000) 209, gr-qc/0004007; S. Tsujikawa, B. Gumjudpai, Phys. Rev. D 69 (2004) 123523, astro-ph/0402185; E. Elizalde, S. Nojiri, S.D. Odintsov, D. Saez, V. Faraoni, arXiv: 0803.1311 [hepth]. 